\title{
Determinants of esophageal varices bleeding in patients with advanced hepatocellular carcinoma treated with sorafenib
}

United European Gastroenterology Journal 2016, Vol. 4(3) 363-370 (C) Author(s) 2015 Reprints and permissions: sagepub.co.uk/journalsPermissions.nav DOI: $10.1177 / 2050640615615041$ ueg.sagepub.com

(S)AGE

\author{
Massimo lavarone ${ }^{1}$, Massimo Primignani ${ }^{1}$, Sara Vavassori ${ }^{1}$, \\ Angelo Sangiovanni ${ }^{1}$, Vincenzo La Mura ${ }^{2}$, Raffaella Romeo ${ }^{1}$ \\ and Massimo Colombo ${ }^{1}$
}

\begin{abstract}
Background and aims: Sorafenib is the standard of care for patients with advanced hepatocellular carcinoma (HCC), yet treatment safety may be challenged by portal hypertension. We therefore assessed the prevalence, risk factors and clinical consequences of esophageal varices (EVs) in sorafenib-treated patients with HCC.

Methods: Starting in 2008, all compensated patients with advanced or intermediate HCC not eligible for other therapies were consecutively enrolled in a prospective evaluation of sorafenib therapy, all with pretreatment by upper-gastrointestinal endoscopy (UGE).

Results: A total of 150 patients received sorafenib for 4.6 (95\% Cl, 3.3-5.6) months. At baseline, 61 (41\%) patients were EV free (group A), 78 (52\%) had EVs (61 small EVs (group B), 17 medium/large EVs (group C)) and 11 (7\%) previously endoscopically treated EVs (group D). Propranolol was given to all patients with medium/large EVs and those with previous bleeding. Twelve patients (8\%) bled from EVs after 36 (18-260) days of sorafenib. During sorafenib, bleeding occurred in six of 26 group B patients with neoplastic portal vein thrombosis (nPVT), three of nine group C patients with nPVT, two of five group D patients with nPVT and one of six without nPVT $(p<0.0001)$, nPVT being the strongest independent predictor of bleeding by multivariate analysis ( $\mathrm{HR}=15.4,95 \% \mathrm{Cl} 1.84-129.6)$.

Conclusion: UGE screening is worthwhile in HCC patients allocated to sorafenib since it identifies patients with EVs at risk of bleeding during therapy, particularly those with nPVT.
\end{abstract}

Keywords

Hepatocellular carcinoma, cirrhosis, varices, portal hypertension, upper gastrointestinal endoscopy, portal vein thrombosis

Received: 2 September 2015; accepted: 7 October 2015

\section{Introduction}

Sorafenib, an oral multikinase inhibitor of the vascular endothelial growth factor (VEGF) and platelet-derived growth factor (PDGF) receptors and rapidly accelerated fibrosarcoma (Raf), is the standard of care for clinically compensated patients with advanced hepatocellular carcinoma (HCC) since it has been shown to significantly improve survival. ${ }^{1,2}$ In view of its antiangiogenetic activity, sorafenib has also resulted in a decrease of portal hypertension associated with cirrhosis, a frequent companion of HCC, as a consequence of an inhibition of portocollateral vascularization. This was clearly shown by experimental studies of portal hypertension, whereby sorafenib administration led to reduced portal hypertension by decreasing splanchnic neovascularization and intrahepatic fibrosis, ${ }^{3-5}$ as well as in three small clinical reports showing portocollateral changes in cirrhotic patients with advanced HCC under sorafenib therapy. ${ }^{6-8}$ At variance with these observations, however, are several reports of episodes of hemorrhage in patients enrolled both in clinical trials

${ }^{1}$ A.M. \& A. Migliavacca Center for Liver Disease, 1st Division of Gastroenterology, Fondazione IRCCS Ca' Granda Maggiore Hospital, University of Milan, Milan, Italy

${ }^{2}$ First Division of Internal Medicine, IRCCS Policlinico San Donato, University of Milan, Milan, Italy

Corresponding author:

Massimo lavarone, 1st Division of Gastroenterology, Fondazione IRCCS Ca' Granda Ospedale Maggiore, Policlinico Università degli Studi di Milano, Via F. Sforza 35, 20122 Milan, Italy.

Email: massimo.iavarone@gmail.com 
and field practice, ${ }^{9-12}$ although most bleeding episodes were mild and occurred at any site besides the gastrointestinal (GI) tract. ${ }^{13}$ According to a recent metaanalysis of randomized trials, all-grade bleeding events occurred in no more than $14 \%$ (708 of 4934) of patients who were treated with either sorafenib or sunitinib, another inhibitor of multiple receptors of tyrosine kinases, for any solid tumor, conferring an incidence rate of $16.7 \%$ and a relative risk of bleeding of $2.0(95 \%$ confidence interval (CI) 1.14-3.49, $p=0.015) .{ }^{1,10,13-15}$ Interestingly, this meta-analysis demonstrated no additive risk of bleeding for patients with $\mathrm{HCC}$ who had cirrhosis, too. The increased risk of bleeding in HCC exposed to sorafenib was confirmed by another metaanalysis: most episodes of bleeding were low grade and no more frequent than those occurring in patients with renal cell carcinoma. ${ }^{16}$ These observations contradicted the results of the registration trial for sorafenib in HCC where the incidence of GI bleeding was similar in both active drug and placebo arms ( $2 \%$ vs $4 \%$ ), but importantly patients were not examined pre-treatment for the presence of esophageal varices (EVs). ${ }^{1}$ The dichotomous effect of sorafenib increasing the risk of bleeding and decreasing portal hypertension in parallel, together with the lack of data on GI bleeding safety in sorafenibexposed cirrhotic patients, led us to investigate the prevalence, risk factors and clinical consequences of EVs in patients undergoing sorafenib treatment in a prospective cohort study, being a study with a control arm unfeasible after sorafenib approval, even if it would better weigh the impact of sorafenib on EV bleeding.

\section{Materials and methods}

\section{Study design}

This is part of a single-center, investigator-driven, prospective, ongoing study to assess the safety and effectiveness of sorafenib in patients with advanced $\mathrm{HCC}$ or with intermediate HCC not eligible for or failing ablative therapies. The study started in July 2008. Part of the result was previously published. ${ }^{17}$ All patients eligible for sorafenib treatment in our center underwent baseline upper-GI endoscopy (UGE) and were subsequently categorized in one of four categories and treated according to the bleeding risk: A) patients without EVs: no prophylaxis provided; B) patients with small (F1) EVs: no prophylaxis provided; C) patients with medium/large (F2-3) EVs and D) patients with past history of variceal bleeding: In both groups prophylaxis with non-selective beta-blockers (NSBBS) was given at the higher tolerated dose, if not already on treatment. These last patients had been previously treated with endoscopic variceal ligation (EBL) and were on regular endoscopic follow-up and/or NSBBS at the maximal tolerated dose. Excluded were patients with bleeding EV within the prior three months, until fully recovered.

\section{Patients}

All patients with advanced-stage $\mathrm{HCC}$, i.e. classified as Barcelona Clinic Liver Cancer (BCLC) stage C and those with a BCLC-B stage who were not eligible for or had failed to respond to locoregional therapies, were consecutively enrolled. ${ }^{18}$ None had received previous systemic therapy. HCC was diagnosed by the criteria of the American Association for the Study of Liver Disease $^{19}$ and staged by abdominal dynamic contrastenhanced computed tomography (CT scan) or gadolinium-enhanced magnetic resonance imaging (MRI). Each patient also underwent chest X-ray/CT scan and bone scanning. Neoplastic portal vein thrombosis (nPVT) was defined at baseline CT scan or MRI as a filling defect, partially or completely occluding the vessel in the portal venous phase, with clear evidence of enhancement during the arterial phase of dynamic imaging. Portal vein thrombosis (PVT) without this clear-cut evidence was considered as non-neoplastic. PVT extension was classified according to Shi et al. ${ }^{20}$

Exclusion criteria were previous liver transplant and those criteria by the Italian Medicines Agency (AIFA), ${ }^{21}$ i.e. an Eastern Cooperative Oncology Group (ECOG) performance status (PS) score $\mathrm{s}^{22}>2$ and clinical decompensation. Cirrhosis was graded according to the ChildPugh classification. ${ }^{23}$ Written informed consent was obtained from each patient according to the ethics committee and the ethics guidelines of the 1975 Declaration of Helsinki, as updated in 2004.

\section{UGE}

All patients underwent UGE within one month before starting sorafenib treatment. All endoscopic procedures were performed by one skilled endoscopist (MP). EVs were classified according to the North Italian Endoscopic Club criteria: size and location ( $\mathrm{F}$, graded $0-3)$, and red wale marks (RWM). ${ }^{24}$

Before starting sorafenib and during treatment, EBL was not considered for primary prophylaxis, while it was assumed as the first treatment choice in case of acute bleeding from EVs. Additional UGE surveillance during treatment with sorafenib was scheduled according to international guidelines, ${ }^{25}$ unless clinically required.

\section{Treatment schedule, dose modification and interruption}

Sorafenib was administered at a dose of $400 \mathrm{mg}$ twice daily following the indications provided by the 
manufacturer. ${ }^{26}$ Dose reduction and drug discontinuation policies are those previously reported. ${ }^{17}$ Briefly, grade 3-4 adverse events (AEs) resulted in dose modification or treatment interruption whenever the AE was clinically relevant; a dose reduction ( $400 \mathrm{mg}$ once daily) or temporary interruption was maintained until the symptoms resolved to grade 1 or 2 according to the guidelines provided by the manufacturer, followed by a re-escalation to the full dose. Dose was also modified at the patient's request in patients showing grade 2 toxicity or whenever a grade $2 \mathrm{AE}$ was judged clinically relevant. Progressive impairment of liver function was another criterion for dose modification or interruption. Therapy was discontinued whenever the patient developed unacceptable toxicity, or radiologic or symptomatic progression of HCC occurred.

\section{Outcome measures}

Safety was assessed in all patients who received at least one dose of sorafenib, and AEs were graded according to the National Cancer Institute's Common Terminology Criteria (version 3.0). ${ }^{27}$ Bleeding from EV was defined according to the Baveno International Consensus Criteria and treated accordingly. ${ }^{25,28-30}$

Overall survival was measured from the date of starting sorafenib therapy until the date of death from any cause or the date of last visit. Patients dying with radiological and/or clinical evidence of tumor progression at any time point were classified as deaths due to HCC progression. By the same token, patients with clinical decompensation like jaundice, encephalopathy or sepsis lacking radiological signs of cancer progression, were classified as deaths due to end-stage liver disease (ESLD). Deaths due to GI hemorrhage, defined according to the Baveno International Consensus Criteria, ${ }^{25,28-30}$ were reported separately.

\section{Statistical analysis}

Data were collected by experienced medical personnel involved in the study using an electronic database. The primary outcomes were assessed by intention to treat, while continuous variables were expressed as mean \pm standard deviation. Categorical variables were analyzed as frequency and percentages. The following baseline features were considered for univariate analysis: age, gender, ECOG-PS, etiology of liver disease, platelet count, albumin, international normalized ratio (INR) and bilirubin levels, alpha-fetoprotein (AFP) level, EV, nPVT and extrahepatic spread. The serum albumin cutoff value of $\geq 4 \mathrm{~g} / \mathrm{dl}$ derives from data of its predictive power of bleeding in a multicenter randomized study in compensated cirrhotic patients. ${ }^{31}$ Data from patients receiving anti-HCC treatment other than sorafenib were censored at the time of entry in the second-line regimen. Survival was analyzed by Kaplan-Meier estimate, and differences in the survival rates were assessed by the log-rank test. Variables with a $p$ value $<0.10$ at univariate analysis were included in the final multivariate analysis model. Cox's proportional-hazard model was used to identify prognostic factors for mortality in a multiple regression analysis. For all analyses, $p \leq 0.05$ was considered statistically significant. All analyses were carried out with Stata 10.0 statistical package (Stata 1944-2007, College Station, TX, USA).

\section{Results}

By September 2013, 150 patients had been consecutively enrolled. Baseline characteristics of the study population are presented in Table 1. Chronic hepatitis $\mathrm{C}$ virus infection was the predominant etiology; 138 patients $(92 \%)$ were graded as Child-Pugh class A, while the 12 Child-Pugh class B patients lacked ascites, clinically overt jaundice and hepatic encephalopathy. At baseline, 67 patients (45\%) had nPVT: one involving segmental branches of the portal vein (PV1), 31 involving either right or left portal vein branches (PV2), 29 involving the main portal trunk (PV3) and six involving the superior mesenteric vein (PV4). No case of benign PVT was identified in our cohort of patients.

Forty-nine $(33 \%)$ patients had extrahepatic spread of the tumor, mainly in the abdominal lymph nodes, lungs and skeleton. Sorafenib was the first treatment modality in $46(31 \%)$ patients. A total of $108(72 \%)$ patients were in the BCLC-C stage and $42(28 \%)$ in the BCLC-B stage, including seven $(17 \%)$ who were not eligible for locoregional treatment. At the time of analysis, $26(17 \%)$ patients were still alive (15 on treatment) and $124(83 \%)$ had died. The median duration of treatment was 4.73 months (95\% CI, 3.45-5.92) and the overall survival was 9.01 months (95\% CI, 6.62-10.32).

At baseline 61 (41\%) patients had no EVs (group A), $61(41 \%)$ patients had small EVs (group B), 17 (11\%) had medium/large EV (group C) and 11 (7\%) previously ligated EVs in secondary prophylaxis (group D), with at least three stable investigations before starting treatment with sorafenib. Among patients with EVs, six had RWM on the variceal wall. All patients with medium/large EVs and those with a previous bleeding were given prophylaxis with NSBBS at the maximal tolerated dose (mean $\pm \mathrm{SD} 80 \pm 15 \mathrm{mg}$; mean \pm SD heart rate $58 \pm 4 / \mathrm{min}$ ) before starting treatment with sorafenib, if not already on treatment.

\section{Bleeding from EVs}

EV bleeding occurred in $12(8 \%)$ patients after 36 (18-260) days of treatment; no different site of bleeding 
Table 1. Baseline demographic, laboratory and clinical characteristics of 150 HCC patients treated with sorafenib

\begin{tabular}{lc}
\hline Patients, no. (\%) & 150 \\
Age, yrs & \\
Male, no. (\%) & $66 \pm 9$ \\
Etiology, no. (\%) & $119(79)$ \\
HCV only & \\
HBV only & $88(59)$ \\
Alcohol abuse only & $27(18)$ \\
Multiple & $18(12)$ \\
Other & $8(5)$ \\
ECOG Performance status, no. (\%) & $9(6)$ \\
0 & \\
1 & $48(32)$ \\
Child-Pugh class, no. (\%) & $102(68)$ \\
A & \\
B & $138(92)$ \\
MELD score, no. (\%) & $12(8)$ \\
$6-8$ & $72(49)$ \\
$\geq 9$ & $77(51)$ \\
Esophageal varices, no. (\%) & \\
Absent & $61(41)$ \\
Small & $61(41)$ \\
Medium/large & $17(11)$ \\
Previously treated & $11(7)$ \\
AFP $\geq 200$ ng/dl, no. (\%) & $24(16)$ \\
Disease burden, no. (\%) & \\
Macroscopic vascular invasion & $67(45)$ \\
Extrahepatic spread & $49(33)$ \\
BCLC score, no. (\%) & $108(72)$ \\
B & $104(69)$ \\
C & \\
Previous treatments, no. (\%) & \\
\hline & \\
\hline
\end{tabular}

a Mean \pm SD. yrs: years; HCC: hepatocellular carcinoma; HCV: hepatitis C virus; HBV: hepatitis B virus; ECOG: Eastern Cooperative Oncology Group; MELD: Model for End-Stage Liver Disease; AFP: alpha fetoprotein; BCLC: Barcelona Clinic Liver Cancer.

was identified in our patients and none had either a recent history of nonsteroidal anti-inflammatory or anticoagulant drug intake. EV hemorrhage occurred in six patients with small EVs without previous bleeding (group B), five with medium/large EVs (group C) and one with previously treated EVs for past history of variceal bleeding (group D). All patients interrupted sorafenib because of the bleeding episode and were treated with emergency EBL and vasoactive drugs. All patients but one permanently discontinued sorafenib after the bleeding episode; two patients underwent a second-line treatment when recovered from bleeding.

\section{Predictors of bleeding from EVs}

By univariate analysis, EV hemorrhage was predicted by albumin level $\leq 4.0 \mathrm{~g} / \mathrm{dl} \quad(p=0.01)$, bilirubin $>1 \mathrm{mg} / \mathrm{dl}$
Table 2. Baseline demographic, laboratory and clinical characteristics of $150 \mathrm{HCC}$ patients according to esophageal varices bleeding occurrence during treatment with sorafenib

\begin{tabular}{|c|c|c|c|}
\hline Characteristics & Bleeders & Non-bleeders & $p$ value \\
\hline Patients, no. (\%) & 12 & 138 & - \\
\hline Age, $y r s^{a}$ & $65 \pm 7.2$ & $66 \pm 9.0$ & 0.64 \\
\hline Male, no. (\%) & $7(58)$ & $112(81)$ & 0.06 \\
\hline HCV positive, no. (\%) & $9(75)$ & $79(57)$ & 0.53 \\
\hline ECOG Performance status 0, no. (\%) & $10(83)$ & $92(67)$ & 0.29 \\
\hline Bilirubin >1.0 mg/dl, no. (\%) & $10(83)$ & $55(40)$ & 0.002 \\
\hline Albumin $\leq 4.0 \mathrm{~g} / \mathrm{dl}$, no. (\%) & $11(92)$ & $63(46)$ & 0.01 \\
\hline Prothrombin time $\geq 1.15$, no. (\%) & $9(75)$ & $67(49)$ & 0.06 \\
\hline $\begin{array}{l}\text { Platelet count } \\
\qquad>100 \times 10^{3} \text { cell } / \mathrm{mm}^{3} \text {, no. (\%) }\end{array}$ & $5(42)$ & $44(32)$ & 0.51 \\
\hline \multicolumn{4}{|l|}{ Esophageal varices, no. (\%) } \\
\hline Absent & $0(-)$ & $61(44)$ & \\
\hline Small & $6(50)$ & $55(40)$ & \\
\hline Medium/large & $3(25)$ & $14(10)$ & 0.0002 \\
\hline Previously treated & $3(25)$ & $8(6)$ & \\
\hline AFP $\geq 100 \mathrm{ng} / \mathrm{dl}$, no. (\%) & $0(-)$ & $25(18)$ & 0.12 \\
\hline Neoplastic PVT, no. (\%) & $11(92)^{\S}$ & $67(49)^{\S}$ & 0.0002 \\
\hline
\end{tabular}

${ }^{a}$ Mean \pm SD. yrs: years; HCC: hepatocellular carcinoma; HCV: hepatitis C virus; HBV: hepatitis B virus; ECOG: Eastern Cooperative Oncology Group; AFP: alpha fetoprotein; PVT: portal vein thrombosis.

( $p=0.002)$, presence of medium/large $(p=0.0002)$ or previously treated EVs $(p=0.02)$ and presence of nPVT $(p=0.0002)$ (Table 2). As shown in Table 3, bleeding on sorafenib occurred in six of 26 group B patients with nPVT, in three of nine group $\mathrm{C}$ patients with nPVT, two of five group D patients with nPVT and one of six without nPVT $(p<0.0001)$. By multivariate analysis, $\mathrm{nPVT}$ (hazard ratio $(\mathrm{HR}=15.82$, 95\% CI 1.90-131.28) and albumin level $\leq 4.0 \mathrm{mg} / \mathrm{dl}$ (HR $0.11,95 \%$ CI $0.14-0.91$ ) were the only independent predictors of bleeding (Table 4).

\section{Survival and outcome measures}

The mean duration of sorafenib treatment was 2.29 months $(0.59-8.57)$ in the 12 patients on treatment for EV bleeding and 6.40 months (0.26-24.16) in those not on treatment for EV bleeding $(p=0.015)$. Among the 12 patients who bled from EVs during sorafenib treatment, at the time of analysis 10 patients had died. Six $(60 \%)$ died of ESLD, two (20\%) of HCC progression and two $(20 \%)$ of EV hemorrhage: the first one, two days after bleeding, the second because of a further bleeding 117 days after sorafenib discontinuation (even though re-bleeding prophylaxis procedures were applied). Among the 138 patients who didn't bleed during sorafenib treatment, at the time of analysis, 114 patients had died: $78(69 \%)$ of HCC progression, 
Table 3. Risk of bleeding according to esophageal varices size and presence of neoplastic portal vein thrombosis in $150 \mathrm{HCC}$ patients treated with sorafenib

\begin{tabular}{llll}
\hline Characteristics & $\begin{array}{l}\text { Bleeders } \\
\text { (No. }=12)\end{array}$ & $\begin{array}{l}\text { Non-bleeders } \\
\text { (No. = 138) }\end{array}$ & $p$ value \\
\hline Group A with nPVT & 0 & 27 & $<0.0001$ \\
Group A without nPVT & 0 & 34 & \\
Group B with nPVT & 6 & 20 & \\
Group B without nPVT & 0 & 35 & \\
Group C with nPVT & 3 & 6 & \\
Group C without nPVT & 0 & 8 & \\
Group D with nPVT & 2 & 3 & \\
Group D without nPVT & 1 & 5 & \\
\hline
\end{tabular}

HCC: hepatocellular carcinoma; EVs: esophageal varices; nPVT: neoplastic portal vein thrombosis; Group A: patients without EVs at baseline; Group B: patients with small EV at baseline; Group C: with medium/large EVs; Group D: patients with past history of variceal bleeding and previously treated with endoscopic variceal ligation.

Table 4. Multivariate analysis of risk factors associated with esophageal varices bleeding in HCC patients treated with sorafenib

\begin{tabular}{lcl}
\hline Predictor (code) & HR (95\% Cl) & $p$ value \\
\hline Bilirubin $>1.0 \mathrm{mg} / \mathrm{dl}$ (yes vs. no) & $2.79(057-13.58)$ & 0.202 \\
Albumin $>4.0 \mathrm{mg} / \mathrm{dl}$ (yes vs. no) & $0.11(0.14-0.92)$ & 0.04 \\
Neoplastic PVT (yes vs. no) & $15.82(1.9-131.28)$ & 0.01 \\
Medium/large EVs (yes vs. no) & $1.99(0.92-4.32)$ & 0.08 \\
Previously treated EVs (yes vs. no) & $1.88(0.88-4.04)$ & 0.10 \\
\hline
\end{tabular}

HCC: hepatocellular carcinoma; EVs: esophageal varices; PVT: portal vein thrombosis; HR: hazard ratio; Cl: confidence interval.

$31(27 \%)$ of ESLD and five (4\%) of EV hemorrhage (19-111 days after sorafenib discontinuation). The overall median survival in the former group was significantly shorter as compared with that of the latter group (4.73 months - 95\% CI, $3.45-5.32$ vs 7.99 months $-95 \%$ CI, 7.99-10.65, $p=0.0035$ ).

\section{Discussion}

Survival benefits emerged in two randomized controlled trials, ${ }^{1,2}$ leading sorafenib to become the standard of care for clinically compensated patients with advanced-stage HCC. However, further emphasizing the peculiarity of HCC and its association with portal hypertension caused by cirrhosis, an increased risk of GI bleeding has been increasingly reported in patients enrolled in sorafenib therapy, possibly as a consequence of the anti-angiogenic activity of the drug, ${ }^{11-13,16}$ together with few data supporting the opposite effect reducing the risk of bleeding. ${ }^{3-8}$ Interestingly, while the rate of bleeding from any source is increased in cancer patients receiving anti-angiogenetic drugs, ${ }^{13,16} \mathrm{EV}$ bleeding was not prominent in patients treated in the registration trial of sorafenib $(2 \%$ in the active drug arm vs $4 \%$ in the placebo arm), ${ }^{1}$ but the lack of UGE studies make the low incidence of variceal bleeding a mere reflection of a low prevalence of EVs in the study population. Moreover, a higher prevalence of patients with significant portal hypertension has been stochastically selected in our clinical practice study as compared to the Sorafenib HCC Assessment Randomized Protocol (SHARP) population, as suggested by different surrogates (i.e. lower median albumin level, higher prevalence of nPVT and slightly higher Child-Pugh and Model for End-Stage Liver Disease (MELD) scores). These AEs on sorafenib, however, are still not perceived important enough to modify pre-treatment patient selection according to the risk of bleeding.

The role of EVs in HCC is not a trivial point, since the increased mortality of HCC patients due to GI bleeding might question the indication for sorafenib treatment on itself. Surprisingly enough, most phase II and III studies testing anti-angiogenic drugs in HCC did not include EV as a pre-treatment selection criterion with the exception of two studies in which UGE was required following life-threatening episodes of GI hemorrhage. ${ }^{1,2,16,32,33}$ To gather more insight into the role of EVs in patients with advanced HCC, we consecutively evaluated all patients suitable for sorafenib treatment and found two-thirds of them having pre-treatment EVs. While one-third of these patients had medium/large varices or a history of bleeding from ruptured EVs, a sizable number of patients had small varices that are known to mark increased bleeding risk, too. Indeed from a prospective study in HCCfree cirrhotics we learned that the two-year risk of bleeding is significantly higher in patients with any grade EVs compared to EV-free patients $(12 \%$ vs $2 \%) .{ }^{34}$ Not surprisingly, therefore, we found GI bleeding during sorafenib treatment to occur in 12 patients $(8 \%)$, in all cases due to ruptured EVs. This is at variance with the prevalence of bleeding in the sorafenib arm of the SHARP study ( $2 \%)$ in contrast with $8 \%$ in our experience, corresponding to $13 \%$, for patients with baseline EVs. In our study EV bleeding was not prevented by propranolol prophylaxis in $24 \%$ of patients with medium-large varices or with a previous bleeding from EVs, which is in line with historical results in HCC-free cirrhotic patients. ${ }^{35,36}$

Before our study, there were few data on the risk of bleeding in untreated advanced HCC patients and little information about the impact of prophylaxis on this population, and a new study in untreated patients is unfeasible after sorafenib approval. In most previous studies of primary and secondary prophylaxis, HCC 
was a criteria for exclusion or at least only patients with early HCC were allowed to be included, thus we were not in a position to fully understand whether strategies of treatment of portal hypertension in HCC-free patients do confer clinical benefits to patients with advanced $\mathrm{HCC}$, eventually on sorafenib treatment.

All in all, our data stress the need that patients with medium-large EV and those previously treated with banding should either undergo a careful clinical surveillance when receiving sorafenib therapy or a different approach should be considered. In our cohort of patients with a past history of variceal bleeding and EBL, we identified only a single HCC patient $(9 \%)$ who bled during sorafenib treatment, with a known risk of re-bleeding in HCC-free cirrhotics between $17 \%$ and $40 \%,{ }^{37}$ suggesting the importance of achieving a good obliteration of EVs before starting sorafenib. However, we should consider-in real life - that the application of portal hypertension therapies in patients with advanced HCC waiting for sorafenib might encounter insurmountable difficulties. These patients, in fact, for whom NSBBS-related AEs can make it difficult to achieve a protective dosage of the drug, can hardly comply with the burden of EBL therapy. The multiple endoscopic sessions required to curb the bleeding risk can be not only unbearable for these frail patients, but can also delay sorafenib treatment, according to the potential for interaction between the drug and the physiological processes of wound healing.

Another interesting finding of our study was the identification of nPVT, whose prevalence is slightly higher than the $36 \%$ of the SHARP study, as a prognostic marker of bleeding. In fact, macroscopic vascular invasion by HCC was detected in 11 of 12 patients who bled, raising the rates of bleeding in patients with medium/large EVs or those previously treated up to $40 \%$, calling for a cautious evaluation of the cost utility ratio of sorafenib therapy in patients with EVs, particularly those with nPVT. A recent retrospective study that compared the outcomes of 146 cirrhotics with HCC who had an acute variceal bleeding with an HCC-free group showed that nPVT was associated with increased rates of five-day death and six-week rebleeding and death. ${ }^{38}$ Overall, PVT, in particular nPVT, was associated with the highest risk of secondary prophylaxis failure (HR: $4.62,95 \%$ CI: $1.96-10.90$ for nPVT). Finally, by multivariate analysis on survival rates of untreated patients in randomized clinical trials of HCC, nPVT emerged as a strong predictor of survival: our finding may suggest a possible explanation of the shortened survival in these patients, even if treated with sorafenib. ${ }^{39}$ We acknowledge that our study has limitations, starting from the obvious drawback of EV bleeding being part of the natural history of cirrhosis independently of HCC. ${ }^{40,41}$ This notwithstanding, our survey allowed us to identify a subset of patients with medium/large or previously treated EVs with nPVT who had an exceedingly high risk of bleeding in the face of a moderate survival benefit conferred by anti-HCC treatment, possibly as a consequence of anticipated interruption of sorafenib.

Interestingly, the reduced survival in patients with variceal bleeding was caused by different reasons (two patients died of bleeding but many died of ESLD), while in the non-bleeders the main cause of death was tumor progression.

With all the caveats of the limited sample size of the study, EVs appear to be a real threat to HCC patients indicated for sorafenib therapy since severe portal hypertension likely accelerates the outcome of HCC patients, raising in parallel the question of whether prophylaxis is more efficacious with endoscopic band ligation or with propranolol. This notwithstanding, we provide here suggestions that screening with UGE patients with advanced HCC prior to starting sorafenib or any other anti-angiogenic drug helps manage the risk of bleeding from EVs.

\section{Funding}

This research received no specific grant from any funding agency in the public, commercial, or not-for-profit sectors.

\section{Conflict of interest}

None declared.

\section{References}

1. Llovet JM, Ricci S, Mazzaferro V, et al. Sorafenib in advanced hepatocellular carcinoma. N Engl J Med 2008; 359: 378-390.

2. Cheng AL, Kang YK, Chen Z, et al. Efficacy and safety of sorafenib in patients in the Asia-Pacific region with advanced hepatocellular carcinoma: A phase III randomised, double-blind, placebo-controlled trial. Lancet Oncol 2009; 10: 25-34.

3. Semela D, Das A, Langer D, et al. Platelet-derived growth factor signaling through ephrin-b2 regulates hepatic vascular structure and function. Gastroenterology 2008; 135: 671-679.

4. Tugues S, Fernandez-Varo G, Muñoz-Luque J, et al. Antiangiogenic treatment with sunitinib ameliorates inflammatory infiltrate, fibrosis, and portal pressure in cirrhotic rats. Hepatology 2007; 46: 1919-1926.

5. Mejias M, Garcia-Pras E, Tiani C, et al. Beneficial effects of sorafenib on splanchnic, intrahepatic, and portocollateral circulations in portal hypertensive and cirrhotic rats. Hepatology 2009; 49: 1245-1256.

6. Coriat R, Gouya H, Mir O, et al. Reversible decrease of portal venous flow in cirrhotic patients: A positive side effect of sorafenib. PLoS One 2011; 6: e16978. 
7. Pinter M, Sieghart W, Reiberger T, et al. The effects of sorafenib on the portal hypertensive syndrome in patients with liver cirrhosis and hepatocellular carcinoma-a pilot study. Aliment Pharmacol Ther 2012; 35: 83-91.

8. Hidaka H, Nakazawa T, Kaneko T, et al. Portal hemodynamic effects of sorafenib in patients with advanced hepatocellular carcinoma: A prospective cohort study. J Gastroenterol 2012; 47: 1030-1035.

9. Kamba T and McDonald DM. Mechanisms of adverse effects of anti-VEGF therapy for cancer. $\mathrm{Br} J$ Cancer 2007; 96: 1788-1795.

10. Motzer RJ, Hutson TE, Tomczak P, et al. Sunitinib versus interferon alfa in metastatic renal-cell carcinoma. $N$ Engl J Med 2007; 356: 115-124.

11. Elice F, Jacoub J, Rickles FR, et al. Hemostatic complications of angiogenesis inhibitors in cancer patients. Am J Hematol 2008; 83: 862-870.

12. Hall PS, Kancherla K, Sastry PS, et al. Severe epistaxis with tyrosine kinase inhibitors. Clin Oncol ( $R$ Coll Radiol) 2008; 20: 318-319.

13. Je Y, Schutz FA and Choueiri TK. Risk of bleeding with vascular endothelial growth factor receptor tyrosinekinase inhibitors sunitinib and sorafenib: A systematic review and meta-analysis of clinical trials. Lancet Oncol 2009; 10: 967-974.

14. Demetri GD, van Oosterom AT, Garrett CR, et al. Efficacy and safety of sunitinib in patients with advanced gastrointestinal stromal tumour after failure of imatinib: A randomised controlled trial. Lancet 2006; 368: 1329-1338.

15. Escudier B, Eisen T, Stadler WM, et al. Sorafenib in advanced clear-cell renal-cell carcinoma. $N$ Engl $J$ Med 2007; 356: 125-134.

16. Duffy A, Wilkerson $\mathrm{J}$ and Greten TF. Hemorrhagic events in hepatocellular carcinoma patients treated with anti-angiogenic therapies. Hepatology 2013; 57: 1068-1077.

17. Iavarone M, Cabibbo G, Piscaglia F, et al. Field-practice study of sorafenib therapy for hepatocellular carcinoma: A prospective multicenter study in Italy. Hepatology 2011; 54: 2055-2063.

18. Llovet J, Brú C and Bruix J. Prognosis of hepatocellular carcinoma: The BCLC staging classification. Semin Liver Dis 1999; 19: 329-338.

19. Bruix J and Sherman M. Management of hepatocellular carcinoma. Hepatology 2005; 42: 1208-1236.

20. Shi J, Lai EC, Li N, et al. Surgical treatment of hepatocellular carcinoma with portal vein tumor thrombus. Ann Surg Oncol 2010; 17: 2073-2080.

21. Agenzia Italiana del Farmaco. Registro farmaci oncologici sottoposti a monitoraggio, http://antineoplastici. agenziafarmaco.it/normativa_registro_trast.htm (accessed January 2015).

22. Oken MM, Creech RH, Tormey DC, et al. Toxicity and response criteria of the Eastern Cooperative Oncology Group. Am J Clin Oncol 1982; 5: 649-655.

23. Pugh RN, Murray-Lyon IM, Dawson JL, et al. Transection of the oesophagus for bleeding oesophageal varices. Br J Surg 1973; 60: 646-649.
24. North Italian Endoscopic Club for the Study and Treatment of Esophageal Varices. Prediction of the first variceal hemorrhage in patients with cirrhosis of the liver and esophageal varices. A prospective multicenter study. N Engl J Med 1988; 319: 983-989.

25. De Franchis R. Evolving consensus in portal hypertension. Report of the Baveno IV consensus workshop on methodology of diagnosis and therapy in portal hypertension. J Hepatol 2005; 43: 167-176.

26. EU summary of product characteristics for Nexavar. Berlin, Germany: Bayer Schering Pharma AG, November 2010.

27. Cancer Therapy Evaluation Program, Common terminology criteria for adverse events, version 3.0, DCTD, NCI, NIH, DHHS, http://ctep.cancer.gov (31 March 2003, accessed 9 August 2006).

28. De Franchis R. Revising consensus in portal hypertension: Report of the Baveno $\mathrm{V}$ consensus workshop on methodology of diagnosis and therapy in portal hypertension. J Hepatol 2010; 53: 762-768.

29. De Franchis R. Developing consensus in portal hypertension. J Hepatol 1996; 25: 390-394.

30. De Franchis R. Updating consensus in portal hypertension: Report of the Baveno III Consensus Workshop on definitions, methodology and therapeutic strategies in portal hypertension. J Hepatol 2000; 33: 846-852.

31. Ripoll C, Groszmann R, Garcia-Tsao G, et al. Hepatic venous pressure gradient predicts clinical decompensation in patients with compensated cirrhosis. Gastroenterology 2007; 133: 481-488.

32. Siegel AB, Cohen EI, Ocean A, et al. Phase II trial evaluating the clinical and biologic effects of bevacizumab in unresectable hepatocellular carcinoma. J Clin Oncol 2008; 26: 2992-2998.

33. Thomas MB, Morris JS, Chadha R, et al. Phase II trial of the combination of bevacizumab and erlotinib in patients who have advanced hepatocellular carcinoma. J Clin Oncol 2009; 27: 843-850.

34. Merli M, Nicolini G, Angeloni S, et al. Incidence and natural history of small esophageal varices in cirrhotic patients. J Hepatol 2003; 38: 266-272.

35. D'Amico G, Pagliaro L and Bosch J. The treatment of portal hypertension: A meta-analytic review. Hepatology 1995; 22: 332-354.

36. Gluud LL and Krag A. Banding ligation versus betablockers for primary prevention in oesophageal varices in adults. Cochrane Database Syst Rev 2012; 8: CD004544.

37. Thiele M, Krag A, Rohde U, et al. Meta-analysis: Banding ligation and medical interventions for the prevention of rebleeding from oesophageal varices. Aliment Pharmacol Ther 2012; 35: 1155-1165.

38. Ripoll C, Genescà J, Araujo IK, et al. Rebleeding prophylaxis improves outcomes in patients with hepatocellular carcinoma. A multicenter case-control study. Hepatology 2013; 58: 2079-2088.

39. Cabibbo G, Enea M, Attanasio M, et al. A meta-analysis of survival rates of untreated patients in randomized clinical trials of hepatocellular carcinoma. Hepatology 2010; 51: 1274-1283. 
40. Amitrano L, Guardascione MA, Bennato R, et al. MELD score and hepatocellular carcinoma identify patients at different risk of short-term mortality among cirrhotics bleeding from esophageal varices. J Hepatol 2005; 42: 820-825.
41. Kadouchi K, Higuchi K, Shiba M, et al. What are the risk factors for aggravation of esophageal varices in patients with hepatocellular carcinoma? J Gastroenterol Hepatol 2007; 22: 240-246. 